\title{
Repeat Treatments for Fertile PGD Couples - When to Quit
}

\author{
Shiri Barbash Hazan MD ${ }^{1}$, Mira Malcov PhD ${ }^{1}$, Veronica Gold MSc ${ }^{1}$, Sagit Peleg PhD ${ }^{1}$, Yael Kalma \\ PhD $^{1}$, Adi Reches MD ${ }^{1,3}$, Hadar Amir MD PhD ${ }^{1}$, Foad Azem MD ${ }^{1}$ and Dalit Ben-Yosef PhD ${ }^{1,2 *}$ \\ ${ }^{1}$ IVF Lab and Wolf PGD Stem Cell Lab, Racine IVF Unit, Lis Maternity Hospital, Tel Aviv Sourasky Medical Center, Israel
}

${ }^{2}$ Department of Cell and Developmental Biology \& Sagol Scholl of Neuroscience, Tel Aviv University, Israel

${ }^{3}$ Genetic Institute, Tel Aviv Sourasky Medical Center, Israel

*Corresponding author: Dalit Ben-Yosef, PhD, Lis Maternity Hospital, Tel Aviv Sourasky Medical Center, 6 Weizmann Street, Tel Aviv 6423906, Israel.

To Cite This Article: Shiri Barbash Hazan MD, Mira Malcov PhD, Veronica Gold MSc, SagitPeleg PhD, DalitBen-YosefPhD, etal., Repeat Treatments for Fertile PGD Couples - When to Quit. Am J Biomed Sci \& Res. 2021 - 13(5). AJBSR.MS.ID.001913. DOI: 10.34297/AJBSR.2021.13.001913.

Received: 監 July 27, 2021; Published: 眥 July 30, 2021

\section{Opinion}

The vast evolution in in vitro fertilization (IVF) and human genetics over the past 30 years made it possible to develop preimplantation genetic diagnosis (PGD) for couples' carriers of genetic diseases. The indications for PGD are genetic disorders with a risk of $\geq 25 \%$ for offspring to be affected by monogenic diseases with recessive, dominant and X-linked inheritances, translocations or other chromosomal aberrations. This technology is wildly accepted as being robust, sensitive and highly accurate, precluding misdiagnosis by allowing the amplification of both the familial mutation and several flanking informative polymorphic markers $[1,2]$, thereby dramatically reducing the risk of misdiagnosis due to allele dropout of the mutated allele [3]. Thus, although these couples are usually fertile, PGD ensures the birth of an unaffected child and avoid pregnancy with affected fetuses that obligate termination of the pregnancy.

The current study was designed to identify variables associated with success and failure of IVF-PGD treatment in fertile patients with a genetic risk, in order to choose for them the preferred treatment. The study was approved by the ethics committee (\#0748/15) and included 148 cycles of 23 women who underwent PGD due to severe genetic conditions that were unsuccessful and conceived spontaneously soon after (within one year) the last failed PGD cycle. The control group included 86 cycles of 63 women who underwent PGD within the same time period and had a successful

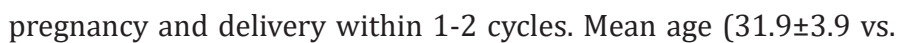

$30.6 \pm 4.5$ years $)$ and history of previous pregnancies $(0.7 \pm 0.8$ vs. $0.8 \pm 0.9$ ), were similar for the study and control groups. The study group had significantly more previous spontaneous pregnancies $(2.3 \pm 1.7$ vs. $1.6 \pm 1.4, \mathrm{p}<0.05)$ and more terminations of pregnancy due to an affected fetus ( $1.6 \pm 1.5$ vs. $0.7 \pm 0.9, p<.05)$, which is indicative of their fertility potential. The women in the study group started significantly more cycles compared to the controls $(6.4 \pm 3.3$ vs. $1.4 \pm 0.5, \mathrm{p}<.05)$, but significantly more of them were cancelled $(0.5 \pm 0.9$ vs. $0, p<.05)$ due to poor ovarian response (Table 1$)$. Altogether, the study group had a mean of $5.9 \pm 3.3$ cycles with no pregnancy, compared to the control group in which each woman became pregnant following only $1.4 \pm 0.5$ cycles $(p<.05)$. Although the women in the study group received a higher dose of FSH analog in each ovarian stimulation IVF-PGD cycle, they responded with lower estradiol blood levels $(\mathrm{p}<.05)$. The progesterone levels and the endometrium thicknesses, however, were not different between the two groups ( $p>05)$ (Table 1). The women in the study group had significantly fewer oocytes and embryos than the control, in each treatment cycle, i.e. they had significantly fewer mature oocytes ( $7.8 \pm 5.7$ vs. $13.1 \pm 7.6$ ), less embryos ( $5.8 \pm 4.5$ vs. $10.1 \pm 5.8$ ), less embryos were biopsied for PGD $(4.0 \pm 3.4$ vs. $7.4 \pm 4.0)$ and thus the number of healthy embryos available for transfer in each cycle was also significantly reduced $(1.5 \pm 1.2$ vs. $2 \pm 0.9 ; \mathrm{p}<0.05)$. The cumulative fertilization and embryo development in all cycles that each woman underwent were compared between groups and the results show that in total, more oocytes were retrieved for each 
women in the study group compared to the control group $(60.3 \pm 58.8$ vs. $21.1 \pm 13, \mathrm{p}<.05)$, more embryos were derived (34.4 \pm 30.8 vs. $13.8 \pm 8.8 \mathrm{p}<.05)$, and more healthy embryos were transferred following biopsy and diagnosis $(8.7 \pm 6.8$ vs. $2.8 \pm 1.5$, $\mathrm{p}<.05$; (Table 2 ). In addition, the women in the study group had fewer unaffected embryos for transfer per cycle than the control group (1.4 \pm 1.2 vs. $3.4 \pm 3.7 ; \mathrm{p}<.05$,). However, when taking into account all PGD cycles that each woman accomplished, we found that those in the study group had more unaffected embryos available for transfer than those in the control group $(9.7 \pm 9.5$ vs. $4.4 \pm 2.4, \mathrm{p}<0.05$; (Table 3 ), but still they failed to conceive. Moreover, the percentage of termination of pregnancies (TOP) in the study group was also high 34\% (15 TOP out of 44 spontaneous pregnancies, with each patient having 1-4 spontaneous pregnancies following PGD failure). All these patients however, when quitting PGD and switching to natural conception, have delivered healthy babies (29 babies born for these 23 patients).

\begin{tabular}{|c|c|c|c|}
\hline & $\begin{array}{l}\text { Study Conceived spontaneously within one year } \\
\text { after the last failed PGD cycle } 148 \text { cycles }\end{array}$ & $\begin{array}{l}\text { Control Successful PGD } 86 \\
\text { cycles }\end{array}$ & p-value \\
\hline Average cycles/woman & $6.4 \pm 3.3$ & $1.4 \pm 0.5$ & S \\
\hline Average cancelled cycles/woman & $0.5 \pm 0.9$ & 0 & S \\
\hline Average cycles with OPU/woman & $5.9 \pm 3.3$ & $1.4 \pm 0.5$ & $S$ \\
\hline FSH analog/cycle (IU/ml) & $3262 \pm 1683$ & $2696 \pm 1376$ & S \\
\hline Estradiol level/cycle (pg/ml) & $1854 \pm 1589$ & $3104 \pm 1819$ & S \\
\hline Progesterone level/cycle (ng/ml) & $1.2 \pm 1.1$ & $1.1 \pm 1.0$ & NS \\
\hline Endometrium thickness/cycle19 & $9.1 \pm 1.7$ & $9.1 \pm 1.9$ & NS \\
\hline
\end{tabular}

Table 2: Cumulative fertilization and embryo development results per woman.

\begin{tabular}{|c|c|c|c|}
\hline & Study & Control & p value \\
\hline OPU/woman & $60.3 \pm 58.8$ & $21.1 \pm 13.0$ & $\mathrm{~S}$ \\
\hline Fertilization rate (\%) & $66.5 \pm 19.5$ & $72.7 \pm 17.0$ & $\mathrm{NS}$ \\
\hline 2PN embryos/woman & $34.4 \pm 30.8$ & $13.8 \pm 8.8$ & $\mathrm{~S}$ \\
\hline Number of biopsied embryos/woman & $23.9 \pm 22.9$ & $10.1 \pm 5.5$ & $\mathrm{~S}$ \\
\hline Biopsied embryos/2PN (\%) & $72.0 \pm 23.5$ & $80.7 \pm 24.7$ & $\mathrm{NS}$ \\
\hline \# of ET/woman & $8.7 \pm 6.8$ & $2.8 \pm 1.5$ & $\mathrm{~S}$ \\
\hline
\end{tabular}

Table 3: Number of disease-free embryos diagnosed by PGD.

\begin{tabular}{|c|c|c|c|}
\hline & Study & Control & p value \\
\hline Per cycle & $1.4 \pm 1.2$ & $3.4 \pm 3.7$ & $\mathrm{~S}$ \\
\hline Per woman & $9.7 \pm 9.5$ & $4.4 \pm 2.4$ & $\mathrm{~S}$ \\
\hline S - Significant $p<.05$ & & \\
\hline
\end{tabular}

\section{In conclusion}

The current study classified women who achieved pregnancy of healthy offspring from PGD within a reasonable number of cycles, and differentiate them from those who conceived spontaneously and underwent invasive prenatal testing (CVS or amniocentesis). Our result show that suboptimal ovarian response to FSH stimulation may assist in switching women from PGD back to spontaneous pregnancy. Having not achieved a pregnancy following PGD, the women in the study group underwent repeated PGD cycles and although having many healthy embryos for transfer they did not conceive. This may indicate a problem that originated from the ovarian stimulation procedure that resulted in a lower oocyte quantity together with inadequate maturity and competence of the oocytes to develop into implanted embryos (i.e., lower oocyte quality). Although not analyzed in this study, we still cannot exclude the possibility that sperm factors can sometimes also contribute to the PGD failure, as may the female age, body mass index, ethnicity, ovarian dysfunction, and broader demographic profiles [4-6]. While the use of IVF has been reported to be suitable for many infertile and PGD couples, a specific group pf patients can fail PGD mainly due to low quality and quantity of oocytes/embryos [7]. During the growth phase of folliculogenesis, the oocyte produces 
and accumulates molecules and organelles that are fundamental for the development of the preimplantation embryo. Ovulation of the oocyte is concomitant to its maturation that is accompanied by completion of the first meiosis and entrance into the second meiosis until arrest at the MII stage. An inability to properly complete the maturation process will lead to meiotic errors that will result in aneuploid oocytes and embryos. This nuclear oocyte maturation is accompanied by cytoplasmic maturation that is crucial for developmental competence.

The results of the present study thus suggest that fertile women with inadequate ovarian response during in vitro fertilization may sometimes not benefit from PGD, and thus spontaneous pregnancy combined with prenatal diagnosis may be preferable for them, despite the added risk of invasive prenatal tests.

\section{References}

1. Dreesen J, Destouni A, Kourlaba G, Birte Degn, Wulf Christensen Mette, et al. (2014) Evaluation of PCR-based preimplantation genetic diagnosis applied to monogenic diseases: a collaborative ESHRE PGD consortium study. Eur J Hum Genet 22(8): 1012-1008.
2. Basille C, Frydman R, El Aly A, Laetitia Hesters, Renato Fanchin, et al. (2009) Preimplantation genetic diagnosis: state of the art. Eur J Obstet Gynecol Reprod Biol 2009; 145: 9-13.

3. Malcov M, Naiman T, Yosef DB, Ariella Carmon, Nava Mey-Raz, et al. (2007) Preimplantation genetic diagnosis for fragile X syndrome using multiplex nested PCR. Reprod Biomed Online 14(4): 515-521.

4. Iglesias C, Banker M, Mahajan N, Leyre Herrero, Marcos Meseguer, et al. (2014) Ethnicity as a determinant of ovarian reserve: differences in ovarian aging between Spanish and Indian women. Fertil Steril 102(1): 244-249.

5. Dayal MB, Gindoff P, Dubey A, Trimble L B Spitzer, Ashlee Bergin, et al. (2009) Does ethnicity influence in vitro fertilization (IVF) birth outcomes? Fertil Steril 91(6): 2414-2418.

6. Bailey AP, Hawkins LK, Missmer SA, Katharine F.Correia, Elena H.Yanushpolsky (2014) Effect of body mass index on in vitro fertilization outcomes in women with polycystic ovary syndrome. Am J Obstet Gynecol 211(2): 163 e1-166e6.

7. Yeste M, Jones C, Amdani SN, Sheena Patel, Kevin Coward (2016) Oocyte activation deficiency: a role for an oocyte contribution? Hum Reprod Update 22(1): 23-47. 